\title{
The blazar-like radio structure of the TeV source IC 310
}

\author{
M. Kadler ${ }^{1}$, D. Eisenacher ${ }^{1}$, E. Ros ${ }^{2,3}$, K. Mannheim ${ }^{1}$, D. Elsässer ${ }^{1}$, and U. Bach ${ }^{3}$ \\ ${ }^{1}$ Lehrstuhl für Astronomie, Universität Würzburg, Campus Hubland Nord, Emil-Fischer-Straße 31, 97074 Würzburg, Germany \\ e-mail: [matthias.kadler; mannheim;elsaesser] @astro.uni-wuerzburg.de; \\ dorit.eisenacher@physik.uni-wuerzburg.de \\ 2 Departament d'Astronomia i Astrofísica, Universitat de València, 46100 Burjassot, València, Spain \\ e-mail: Eduardo.Ros@uv.es \\ 3 Max-Planck-Institut für Radioastronomie, Auf dem Hügel 69, 53121 Bonn, Germany \\ e-mail: ubach@mpifr.de
}

Received 6 October 2011 / Accepted 23 December 2011

\begin{abstract}
Context. The radio galaxy IC 310 in the Perseus cluster has recently been detected in the gamma-ray regime at $\mathrm{GeV}$ and $\mathrm{TeV}$ energies. The TeV emission shows time variability and an extraordinarily hard spectrum, even harder than the spectrum of the similar nearby gamma-ray emitting radio galaxy M 87.

Aims. High-resolution studies of the radio morphology help to constrain the geometry of the jet on sub-pc scales and to find out where the high-energy emission might come from.

Methods. We analyzed May 2011 VLBA data of IC 310 at a wavelength of $3.6 \mathrm{~cm}$, revealing the parsec-scale radio structure of this source. We compared our findings with more information available from contemporary single-dish flux density measurements with the 100-m Effelsberg radio telescope.

Results. We have detected a one-sided core-jet structure with blazar-like, beamed radio emission oriented along the same position angle as the kiloparsec scale radio structure observed in the past by connected interferometers. Doppler-boosting favoritism is consistent with an angle of $\theta \lesssim 38^{\circ}$ between the jet axis and the line-of-sight, i.e., very likely within the boundary dividing low-luminosity radio galaxies and BL Lac objects in unified schemes.

Conclusions. The stability of the jet orientation from parsec to kiloparsec scales in IC 310 argues against its classification as a headtail radio galaxy; i.e., there is no indication of an interaction with the intracluster medium that would determine the direction of the tail. IC 310 seems to represent a low-luminosity FRI radio galaxy at a borderline angle to reveal its BL Lac-type central engine.
\end{abstract}

Key words. galaxies: active - techniques: interferometric - X-rays: galaxies - radio continuum: general - gamma rays: galaxies galaxies: individual: IC 310

\section{Introduction}

The S0 galaxy IC 310 (B0313+411, J0316+4119) is located in the Perseus Cluster at a redshift of $z=0.0189$. Due to its kiloparsec-scale radio morphology, it has been classified as a head-tail or, more specifically, narrow-angle tail radio galaxy (see Sijbring et al. 1998; and Feretti et al. 1998). The radio emission extends up to a distance of projected $400 \mathrm{kpc}$. Characteristic for the head-tail radio galaxies, which are found only in clusters of galaxies, is that the jet direction is determined by the galaxy's motion through the intracluster medium. The "head" then marks the bow shock thanks to the impact of the jet on the intracluster medium, and the tail corresponds to the redirected extended jet. Along with NGC 1265, IC 310 was in fact the first source classified as a tailed radio galaxy (Ryle \& Windram 1968) since its narrow, elongated large-scale radio structure roughly points radially away from the center of the Perseus Cluster.

The MAGIC collaboration (Mariotti 2010) reported the discovery of very high-energy (VHE) gamma-rays at photon energies $E>100 \mathrm{GeV}$ from IC 310 between October 2009 and February 2010. Neronov et al. (2010) reported on a Fermi/LAT detection above $30 \mathrm{GeV}$ and the source is included in the Fermi Large Area Telescope second source catalog (The FermiLAT Collaboration 2011a), as well as in the second catalog of active galactic nuclei (AGN) detected by the Fermi Large Area Telescope (The Fermi-LAT Collaboration 2001b). The origin of the gamma-ray emission has remained elusive, with the putative bow shock, the jet, or the core considered as the possible sites. The VHE spectral slope is extremely hard (2.00 \pm 0.14$)$ and the source shows gamma-ray variability (Aleksic et al. 2010).

IC 310 is the fourth closest AGN detected so far at VHE gamma-rays, after Centaurus A $(z=0.00183)$, M $87(z=0.004)$, and 3C 84 (a.k.a. as NGC 1275; $z=0.017559$, belonging to the Perseus cluster as well, and also detected by Fermi/LAT). M87 in the Virgo Cluster shares many properties similar to IC 310, and here the emission could be traced to a blazar-like central engine (Aharonian et al. 2006; Acciari et al. 2009). Unification models of radio-loud active galactic nuclei predict low-luminosity FRI radio galaxies to be the parent population of BL Lacertae objects whose characteristics would only become dominant for small jet angles to the line-of-sight, although other effects (e.g., hidden emission line regions, adiabatic losses, mass entrainment, jet deceleration, spine-sheath structure) complicate the picture (Xu et al. 2000).

Throughout this Letter, we use the present standard cosmological model for which 1 arcsec in the sky corresponds to a distance of $0.361 \mathrm{kpc}$ at the distance of IC 310 (see Wright 2006). 
Table 1. Effelsberg flux density measurements.

\begin{tabular}{lcccccc}
\hline \hline Band & $\begin{array}{c}\lambda \\
{[\mathrm{cm}]}\end{array}$ & $\begin{array}{c}v \\
{[\mathrm{GHz}]}\end{array}$ & $\begin{array}{c}I \\
{[\mathrm{mJy}]}\end{array}$ & $\begin{array}{c}P \\
{[\mathrm{mJy}]}\end{array}$ & $\begin{array}{c}m \\
{[\%]}\end{array}$ & $\begin{array}{c}\chi \\
{[\mathrm{deg}]}\end{array}$ \\
\hline $\mathrm{S}$ & 11 & 2.64 & $383 \pm 3$ & $17 \pm 4$ & $4.5 \pm 1.1$ & $-2 \pm 7$ \\
$\mathrm{C}$ & 6 & 4.85 & $238 \pm 4$ & $10 \pm 4$ & $4.0 \pm 1.7$ & $-29 \pm 9$ \\
$\mathrm{X}$ & 3.6 & 8.35 & $148 \pm 3$ & $<10$ & $\ldots$ & $\ldots$ \\
$\mathrm{X}$ & 2.8 & 10.45 & $155 \pm 15$ & $<10$ & $\ldots$ & $\ldots$ \\
$\mathrm{U}$ & 2 & 14.60 & $103 \pm 6$ & $\ldots$ & $\ldots$ & $\ldots$ \\
\hline
\end{tabular}

\section{Observations}

\subsection{Effelsberg}

Motivated by the high-energy detection of the putative headtail radio galaxy IC 310 (Mariotti 2010), we performed a run of single-dish test observations with Effelsberg on July 23, 2011 at five frequencies between $2.64 \mathrm{GHz}$ and $14.60 \mathrm{GHz}$ (see Table 1). We cross-scaned the source, together with some primary and secondary calibrators (namely, 3C 286, NGC 7027, and 3C 48). Depending on the wavelength the integration times varied from $20 \mathrm{~s}$ to $60 \mathrm{~s}$ with longer integrations at shorter wavelengths. Our results are presented in Sect. 3.1.

\subsection{VLBA}

We have processed archival data (experiment codes BC196Q and BC196R) from the Very Long Baseline Array (VLBA) taken at a wavelength of $3.6 \mathrm{~cm}$ on May 16 and 30, 2011, respectively. Those data were correlated at the NRAO Array Operations Center using the DiFX software corrrelator (see Deller et al. 2011). Our target source was observed during $5 \mathrm{~min}$ at each observing run for a program to observe 2MASS galaxies (Condon et al. 2011). Data were recorded with a ultra-wide frequency setup, using eight sub-bands of $16 \mathrm{MHz}$ each spread over a range from $7.9 \mathrm{GHz}$ to $8.9 \mathrm{GHz}$, with a data bit rate of $512 \mathrm{Mbps}$. After downloading the data from the archives, we performed the phase and amplitude calibration using standard methods within the AIPS software package. We used the measurements of system temperatures and the gain curves of the stations to calibrate the visibility amplitude (task APCAL), after doing the digital sampling correction (task ACCOR). We corrected for the instrumental phase and delay offsets using pulse-calibration data recorded during the observations at each antenna (task PCCOR). We obtained the group-delay and phase-rate calibration for each sub-band from a fringe search (task FRING) We got a high detection rate so that no phase-referencing was needed to detect our target source. The accumulation time used at the correlator was of $0.25 \mathrm{~s}$, but we averaged the data to $4 \mathrm{~s}$ segments without loss of information or time smearing.

\section{Results}

\subsection{Effelsberg radio spectrum}

From the cross-scan observations, source flux densities were determined following the procedures described by Kraus et al. (2003). From $2.64 \mathrm{GHz}$ to $10.45 \mathrm{GHz}$, the measured full width half maximum (FWHM) in elevation was somewhat larger than the nominal antenna beam width, indicating that the source was marginally resolved along the main jet axis (compare Feretti et al. 1998, for an Effelsberg map of IC 310 at $10.6 \mathrm{GHz}$ ). At the highest frequency of $14.6 \mathrm{GHz}$ IC 310 appeared compact. The results are shown in Table 1, displaying the name of the band, the observing wavelength and frequency, total flux density $I$, linearly polarized flux density $P$, polarization degree $m$, and electric vector position angle $\chi$.

IC 310 exhibited a steep radio spectrum with the flux density $S$, which is described well by a power law $S \propto v^{\alpha}$ with a spectral index $\alpha=-0.75$. From previous studies with the Westerbork and Effelsberg telescopes (Sijbring \& de Bruyn 1998; Feretti et al. 1998), it is known that this behavior comes from the optically thin emission of the kiloparsec-scale radio jet of IC 310, which dominates the spectrum at low frequencies.

At $8.3 \mathrm{GHz}$ (the frequency of the VLBA observations), we find a total radio flux density of $(148 \pm 3)$ mJy. A comparison with the total flux density emitted on parsec scales and recovered by the VLBA observations (see below) shows that $>80 \%$ of the emission at this high frequency is created on scales smaller than 1 milliarcsec.

\subsection{VLBA imaging results}

After AIPS processing, we exported our data into the software package DIFMAP, where we performed hybrid mapping using the CLEAN algorithm. Since the a priori amplitude calibration was excellent, as seen in other calibrators used during the observations (i.e., NGC 1052, 0059+581, 2200+420), we started our processing by only phase self-calibration. During the imaging process, we tested and applied different weighting schemes (natural weighting, uniform weighting, and natural weighting with downweighting the longest baselines, tapering). Because of the sparse $(u, v)$-coverage, we restricted the CLEAN-region to an area of 8 to 10 beam sizes to keep the number of CLEAN components small, hence ensuring that the number of degrees of freedom can be supported by the data. In all cases we obtained a core-jet morphology with extended emission oriented towards the southeast with a position angle of $-135^{\circ}$, reaching up to 20 mas and with hints of emission up to 40 mas away from the brightest component. The images resulting from this process, with natural weighting and full resolution, are presented in Fig. 1.

We continued our analysis by applying gentle amplitude selfcalibration (typical corrections are smaller than 10\%). This, together with the short time observed (5.3 min each time) makes the quality of the images quite remarkable, with a dynamic range of $\sim 150: 1$.

Both data sets on May 16 and May 30 had similar flux density measured at the shortest VLBA baseline between Los Alamos and Pie Town. Comparing the two independent images, we find that no significant variability occurred between both epochs, so we merged both 5-min data sets (AIPs task DBCON) to get an image with a higher signal-to-noise ratio. In Fig. 2, we show the results of hybrid mapping with a Gaussian tapering at $125 \mathrm{M} \lambda$, together with an image of the NRAO VLA Sky Survey (NVSS; Condon et al. 1998) taken on December 1993 with a resolution of $45 \mathrm{arcsec}$. The resulting VLBA beam size projected along the jet direction is $\sim 2$ mas, whereas the resolution obtained with natural weighting and considering all baselines was $\sim 1$ mas in size. The expected thermal noise for the full VLBA at $512 \mathrm{Mbps}$ for $5.3(10.6) \mathrm{min}$ is of $0.229(0.162) \mathrm{mJy} /$ beam $(1-\sigma$ level). Our formal image root-mean-square ( $\mathrm{rms}$ ) noise level reaches these theoretical values (see Table 2) but does not exceed them significantly, verifying that no overcleaning occurred.

Gaussian model fitting the interferometric visibilities of all three data sets yields a core flux density emission of 80-90 mJy, and a fitted size of (0.3-0.4) mas, which corresponds to a brightness temperature on the order of $1.5 \times 10^{10} \mathrm{~K}$. The jet 

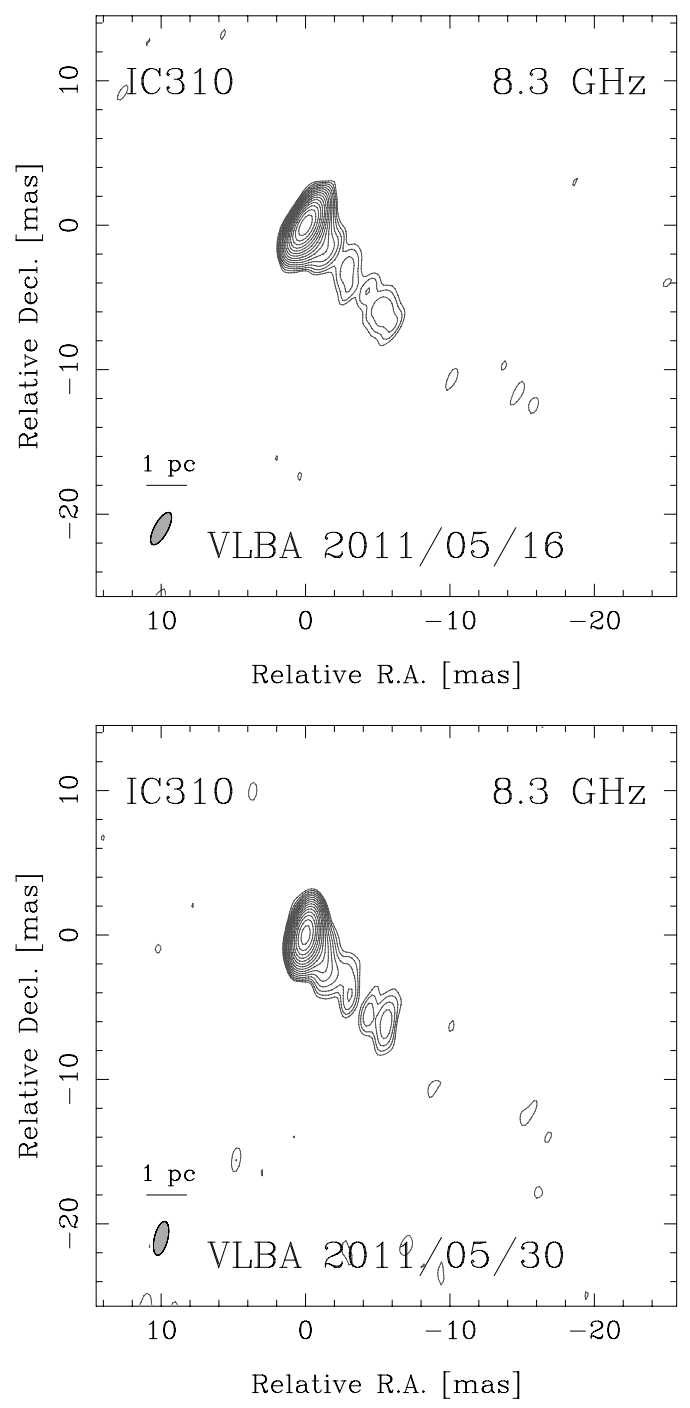

Fig. 1. VLBA images from IC 310 taken on May 16 (top) and May 30 (bottom), 2011. The image parameters are given in Table 2. Lowest contours are $2.5 \times$ the rms level.

Table 2. VLBA map parameters.

\begin{tabular}{lcccc}
\hline \hline Epoch & $\begin{array}{c}S_{\text {tot }} \\
{[\mathrm{mJy}]}\end{array}$ & $\begin{array}{c}S_{\text {peak }} \\
{[\mathrm{mJy} / \mathrm{beam}]}\end{array}$ & $\begin{array}{c}\mathrm{rms} \\
{[\mathrm{mJy} / \mathrm{beam}]}\end{array}$ & $\begin{array}{c}\text { Beam } \\
{[\mathrm{mas} \times \mathrm{mas}, \mathrm{deg}]}\end{array}$ \\
\hline $2011 / 05 / 16$ & $118.8 \pm 6.0$ & $88.7 \pm 4.4$ & 0.20 & $2.50 \times 0.94,-28.4$ \\
$2011 / 05 / 30$ & $115.0 \pm 6.1$ & $85.1 \pm 4.6$ & 0.21 & $2.39 \times 0.94,-13.5$ \\
Comb. & $115.6 \pm 7.9$ & $84.8 \pm 5.7$ & 0.16 & $2.34 \times 0.93,-21.5$ \\
Comb. Taper & $113.6 \pm 7.7$ & $94.5 \pm 6.4$ & 0.17 & $2.63 \times 1.76,-21.5$ \\
\hline
\end{tabular}

components are responsible for additional $14 \mathrm{mJy}$ at a distance of $0.8 \mathrm{mas}$ in $\mathrm{PA}-144^{\circ}$, and then an additional $10 \mathrm{mJy}$ in the range of 3 mas to 8 mas away from the core; i.e., the compactness of the source from the $8.3 \mathrm{GHz}$ snapshot images is $\sim 70 \%$.

\section{Discussion}

Figure 2 shows that the parsec-scale jet obtained with the VLBA snapshot images and the kiloparsec-scale jet from the NVSS are oriented in the same direction. That is, there is no misalignment over several orders of magnitude in the scale of the jet. In more powerful and more distant sources, the one-sidedness of jets is usually attributed to relativistic bulk motion along a relatively small angle to the line of sight, which leads to Doppler boosting of the jet and deboosting of the counterjet emission. With no evidence of any counterjet emission on any scale, we can assume that the peak signal at $8.3 \mathrm{GHz}$ recovered by the VLBA may be attributed to the jet and that the counterjet signal is suppressed to a value below $3 \sigma$, with $\sigma$ the root-mean-square noise in the final VLBA image. Under these assumptions, the jet-to-counterjet ratio is $R>177$. Assuming now that Doppler-boosting is responsible for the one-sidedness, which is generally considered to be the main factor on the pc-scale in unified models for radio galaxies and BL Lacs, $R$ is related to the jet speed $\beta$ and the angle to the line of sight $\theta$ by

$R=\left(\frac{1+\beta \cos \theta}{1-\beta \cos \theta}\right)^{2-\alpha}=\left(\beta_{\mathrm{app}}^{2}+\delta^{2}\right)^{2-\alpha}$,

with the spectral index $\alpha$ defined through $S \propto v^{\alpha}$, the apparent jet speed $\beta_{\text {app }}=v_{\text {app }} / c$, and the Doppler factor $\delta$ (Urry \& Padovani 1998). Further studies will measure $\beta_{\text {app }}$ and elucidate if freefree absorption or adiabatic losses can also play a role in this source. In the case of Doppler-boosting favoritism, the measured limit on the jet-to-counterjet ratio leads to an upper limit on $\theta$ :

$\theta<\arccos \left(\frac{R^{\frac{1}{2-\alpha}}-1}{R^{\frac{1}{2-\alpha}}+1}\right) \quad \beta^{-1}$.

Attributing all of the $8.3 \mathrm{GHz}$ VLBA peak flux density to the jet, assuming $\alpha=0$ (for flat-spectrum compact emission), and letting $\beta \rightarrow 1$ lead to $\theta<31^{\circ}$. A somewhat more conservative estimate is derived from using the emission maximum 3 arcmin down the jet seen in the $49 \mathrm{~cm}$ WSRT image of Sijbring et al. (1998). Knowing now that IC 310 shows a one-sided jet morphology on all scales, this local peak of $\sim 400 \mathrm{mJy}$ compared to the $3 \sigma$ noise on the opposite side of the core of $\sim 0.8 \mathrm{mJy}$ leads to a jet-to-counterjet ratio of $R>500$. Using the spectral index $\alpha=-0.75$ measured at this position, the angle to the line of sight is constrained to $\theta<36^{\circ}$. A third way to constrain the jet angle to the line of sight is to consider the first jet maximum in the NVSS image at $1.4 \mathrm{GHz}$ located at about $75^{\prime \prime}(\sim 23 \mathrm{kpc}$ ) downstream from the core of the jet (see Fig. 2). Here, we derive a jet-to-counterjet ratio of 162/0.8 $=202$. With a spectral index of -0.5 at this position, the angle to the line of sight is constrained to $\theta<38^{\circ}$. For these three scenarios $(R>177, \alpha=0 ; R>500, \alpha=-0.75 ; R>202, \alpha=-0.5)$ and an assumed apparent jet speed of $\beta_{\text {app }}=1$, which would be typical of low-luminosity high-peaked BL Lac objects (Piner \& Edwards 2004; Piner et al. 2010), Eq. (1) yields minimum Doppler factors of $\delta>3.5, \delta>2.9$, and $\delta>2.7$, respectively. Thus, the Doppler factor of IC 310 may be well above the typical values estimated for Cen A (e.g., Abdo et al. 2010: $\delta<3.8$ ), M 87 (e.g., Abdo et al. 2009: $\delta \sim 3.9$ ), or NGC 1275 (e.g., Abdo et al. 2009: $\delta \sim 2.3$ ). Based on the available data, the jet morphology and the jet-to-counterjet ratio point towards a blazar-like nature for IC 310, rather than radio-galaxy characteristics, although higher-signal-to-noise-ratio VLBI observations are needed to put stronger constraints on both $R$ and $\delta$.

The blazar-like nature of IC 310 is supported by the findings of Owen et al. (1996) and Rector et al. (1999). In their optical spectroscopic survey of 190 objects in the Abell clusters, the former find four sources with weak nonthermal activity, which they identify as BLLac candidates. IC 310 is one of these four objects. The difficulty of detecting the optical BL Lac in nearby low-luminosity objects like IC 310 is due to the dominance of the host-galaxy star light. At X-ray energies, Sato et al. (2005) 


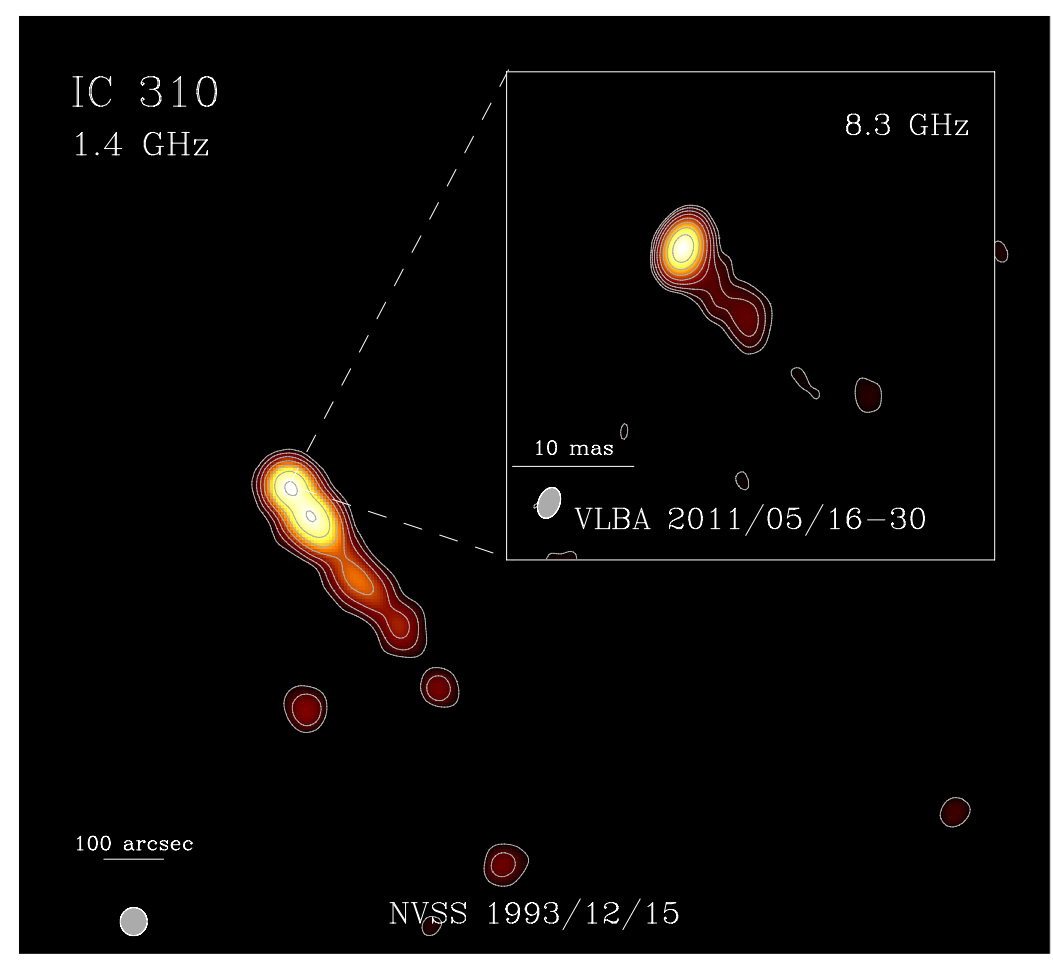

Fig. 2. The jet in IC 310 on small and large scales. Shown are the NVSS image (large field; VLA 1.4 GHz, December 15th, 1993) and the tapered VLBA image (inset panel; 7.9-8.8 GHz, combining data from May 16 and May 30, 2011). The convolving beam of the NVSS image is $45 \times$ 45 arcsec. The lowest contour and the brightness peak of the NVSS image are $3.5 \mathrm{mJy}^{\text {beam }}{ }^{-1}$ and $173.7 \mathrm{mJy}_{\text {beam }}^{-1}$, respectively. Image parameters for the VLBA image are given in Table 2. The lowest contour of the VLBA image is $2.5 \times$ the rms value. find a featureless power-law spectrum from an unresolved point source at the position of the IC 310 nucleus (embedded in strong extended thermal cluster emission) with a rather steep photon index of $\Gamma=2.5$ and suggest a possible BL Lac type nature of this emission as well, although no variability at X-rays has so far been reported. The two-point spectral index between the VHE flux and the (noncontemporaneous) X-ray flux $\alpha_{\mathrm{X} / \mathrm{VHE}} \sim 1$ is also in line with an underlying blazar-type spectral energy distribution (Albert et al. 2008).

\section{Conclusions}

We analyzed archival snapshot $8.3 \mathrm{GHz}$ observations from the VLBA taken in May 2011, and found a core-jet subparsec scale structure in the emission of IC 310. The source is coredominated, with a flux density of around $0.1 \mathrm{Jy}$, and there is extended emission towards the $\mathrm{SE}$, in $\mathrm{PA}-135^{\circ}$, at the same direction of the kiloparsec-scale emission as observed by the VLA in the NVSS survey (Condon et al. 1998) or the WSRT (Feretti et al. 1998).

Single-dish continuum spectra from the 100-m telescope in Effelsberg taken in late July 2011 reveal radio emission at the level of $0.1 \mathrm{Jy}$ at $15 \mathrm{GHz}$ to $0.4 \mathrm{Jy}$ at $2.6 \mathrm{GHz}$. The value of the flux density at the VLBA observing frequency is $\sim 0.15 \mathrm{Jy}$, which implies that the extended emission not detected with the VLBA is smaller than $25 \%$, provided that no significant radio variability at this frequency occurred between May and July 2011.

There is no evidence of interactions of the kpc jet with the intercluster medium as responsible for its narrow-tail morphology; instead, we found evidence that its orientation is inherited from the pc-scale inner jet. It therefore follows that IC 310 should not be classified as a head-tail radio galaxy.

The orientation angle of the jet is consistent with a lowluminosity radio galaxy at the borderline to BL Lacs assuming relativistic bulk motion. This finding corroborates evidence that the high-energy emission of IC 310 originates in the central blazar-like engine.

Acknowledgements. We thank Stefan Rügamer and A.P. Lobanov for valuable discussions and critical reading of the manuscript. We also thank the referee for constructive suggestions. The Very Large Array and the Very Long Baseline Array are operated by the National Radio Astronomy Observatory, a facility of the National Science Foundation operated under cooperative agreement by Associated Universities, Inc. This work made use of the Swinburne University of Technology software correlator, developed as part of the Australian Major National Research Facilities Programme and operated under licence. The results presented here are based on observations with the 100-m telescope of the MPIfR (Max-Planck-Institut für Radioastronomie) at Effelsberg. This research made use of the NASA/IPAC Extragalactic Database (NED), which is operated by the Jet Propulsion Laboratory, California Institute of Technology, under contract with the National Aeronautics and Space Administration. This research made use of NASA's Astrophysics Data System. D.E. acknowledges support by the German BMBF Verbundforschung, and E.R. acknowledges partial support by the Spanish MICINN through grant AYA2009-13036-C02-02, and by the COST action MP0905 "Black Holes in a Violent Universe".

\section{References}

Abdo, A. A., Ackermann, M., Ajello, M., et al. 2009a, ApJ, 707, 55 Abdo, A. A., Ackermann, M., Ajello, M., et al. 2009b, ApJ 699, 31 Abdo, A. A., Ackermann, M., Ajello, M., et al. 2010, ApJ, 719, 1433 Acciari, V. A., Aliu, E., Arlen, T., et al. 2009, Science, 325, 444

Aharonian, F., Akhperjanian, A. G., Bazer-Bachi, A. R., et al. 2006, Science, 314,1424

Albert, J., Aliu, E., Anderhub, H., et al. 2008, ApJ, 681, 944

Aleksić, J., Antonelli, L. A., Antoranz, P., et al. 2010, ApJ, 723, L207

Condon, J. J., Cotton, W. D., Greisen, E. W., et al. 1998, AJ, 115, 1693

Condon, J. J., Darling, J., Kovalev, Y. Y., \& Petrov, L. 2011 [arXiv: 1110.6252]

Deller, A. T., Brisken, W. F., Phillips, C. J., et al. 2011, PASP, 123, 901, 275

Feretti, L., Giovannini, G., Klein, U., et al. 1998, A\&A, 331, 475

The Fermi-LAT Collaboration 2011a, ApJS, submitted [arXiv: 1108.1435]

The Fermi-LAT collaboration 2011b, ApJS, in press [arXiv: 1108.1420]

Kraus, A., Krichbaum, T. P., Wegner, R., et al. 2003, A\&A, 401, 161

Mariotti, M. 2010, ATel, 2510

Neronov, A., Demikoz, D., \& Vovk, Ie. 2010, A\&A, 519, L6

Owen, F. N., Ledlow, M. J., \& Keel, W. C. 1996, AJ, 111, 53

Piner, B. G., \& Edwards, P. G. 2004, ApJ, 600, 115

Piner, B. G., Pant, N., \& Edwards, P. G. 2010, ApJ, 723, 1150

Rector, T. A., Stocke, J. T., \& Perlman, E. S. 1999, ApJ, 516, 145

Rhee, G., Burns, J. O., \& Kowalski, M. P. 1994, AJ, 108, 1137

Ryle, M., \& Windram, M. D. 1968, MNRAS, 138, 1

Sato, K., Furusho, T., Yamasaki, N. Y., et al. 2005, PASJ, 57, 743

Sijbring, D., \& de Bruyn, A. G. 1998, A\&A, 331, 901

Urry, C. M., \& Padovani, P. 1995, PASP, 107, 803

Wright, E. L. 2006, PASP, 118, 1711

Xu, C., Baum, S., O’Dea, C., Wrobel, J. M., \& Condon, J. J. 2000, AJ, 120, 2950 